For further information and registration, contact Workshop on Engineering Controls for Preventing Airborne Infections in Workers in Healthcare Facilities, Project Coordinator (R-2), NIOSH, 476 Columbia Parkway, Cincinnati, OH 45226. Telephone (513) 841-4321.

\section{Drug-Resistant TB Reinfects Same Patient}

Exogenous reinfection with multidrug-resistant M ycobacterium tuberculosis can occur during therapy for the original infection or after therapy has been completed. This finding was reported by Dr. Peter Small and colleagues at the Howard Hughes Medical Institute, Stanford University, San Francisco General Hospital, and the State University of New York Health Science Center at Brooklyn, New York.

The researchers studied 17 patients seen at a New York City hospital who had positive cultures for $M$ tuberculosis repeatedly. Analysis of restrictionfragment-length polymorphisms (RFLP) was performed on serial isolates of $M$ tuberculosis obtained from these patients. Six patients had isolates that remained drug susceptible, and the RFLP patterns of these isolates did not change over time. Eleven patients had isolates that became resistant to antimicrobial agents. The RFLP patterns of the isolates from six of these patients remained essentially unchanged despite the development of drug resistance. In five other patients, however, the RFLP patterns of the isolate changed dramatically at the time that drug resistance was detected. The change in the RFLP pattern of the isolate from one patient appeared to be the result of contamination during processing in the laboratory. In the remaining four patients, all of whom had advanced HIV disease, the clinical and microbiologic evidence was consistent with the presence of active tuberculosis caused by a new strain of $M$ tuberculosis.

The authors concluded that resistance to antituberculosis drugs can develop not only from a mutation in the strain that caused the initial disease, but also as a result of reinfection with a new strain of $M$ tuberculosis that is drug resistant. Thus, exogenous reinfection with multidrug-resistant $M$ tuberculosis can occur either during therapy for the original infection or after therapy has been completed.

The possibility that persons previously infected with $\mathrm{M}$ tuberculosis can be exogenously reinfected has been debated for decades. However, it was thought to occur rarely because of the immunity conferred by the initial infection. On the few occasions in which exogenous reinfection has been documented, it has involved only selected populations, for example, alcoholic residents of a homeless shelter.

The authors note that the lack of acquired immunity to $M$ tuberculosis, which permitted reinfection with a multidrug-resistant strain, presumably would predispose HIV-infected persons to reinfection with drug- sensitive organisms as well. Whether normal hosts can be exogenously reinfected-and if so, how frequently-has not been determined. The lack of protective immunity to $M$ tuberculosis after therapy in HIV-infected patients complicates efforts to control the disease and suggests that those with a history of tuberculosis or known tuberculosis reactivity need to be evaluated for the possible development of a new episode of tuberculosis after contact with someone who is infectious.

It is not possible to infer from this study the frequency with which patients are reinfected with $M$ tuberculosis. The authors note that future studies are needed to define the frequency, settings, and specific risk factors for exogenous reinfection.

FROM: Small PM, Shafer RW, Hopewell PC, et al. NEngl J M ed 1993;328:1137-1 144.

\section{Previous Hospitalization a Risk Factor for Drug-Resistant TB}

Almost $30 \%$ of patients hospitalized in New York City with pulmonary tuberculosis were found to have multidrug-resistant (MDR) disease, and previous hospitalization was found to be a significant risk factor. These findings were reported by Dr. Craig Keyes and colleagues of the New York State Health Department in a paper presented at the May 16-19, 1993, American Lung Association International Conference in San Francisco. Keyes studied sputum samples and demographic and clinical data on 393 patients hospitalized for pulmonary tuberculosis at New York City's 18 metropolitan hospitals. Of the 142 M ycobacterium tuberculosis culture-positive patients, 41 (29\%) were resistant to isoniazid and at least one other standard antituberculosis drug. A number of factors were positively correlated with MDR-TB, including HIV seropositivity, homelessness, Caucasian race, female gender, incarceration, shelter living, and drug or alcohol abuse. However, only previous hospitalization emerged as a significant risk factor for MDRTB. 